\title{
Laboratory diagnosis of infectious diseases: a call for more rapid and accurate methods
}

Before the emergence of antibiotics and vaccines, infectious diseases represented a great threat to human society. Nowadays, high vaccination coverage has helped to significantly reduce the incidence of many infectious diseases. For infected patients, especially those who are infected with bacterial diseases, clinicians can choose from a wide range of antibiotics. The achievements in the development of vaccines and antibiotics have caused many to feel that the war against infectious diseases is winnable. However, the brutal fact is that several novel infectious diseases have emerged within the first 20 years of the 21 st century including severe acute respiratory syndrome (SARS), middle east respiratory syndrome (MERS), and, recently, the 2019 novel coronavirus (2019-nCoV). The emergence of these novel infectious diseases reminds us that there is a long way to go in the war against infectious diseases.

Timely and accurate diagnosis is the first essential step in the management of infectious diseases. Traditionally, microbiological approaches, such as culture and staining, have been the gold standards for diagnosing infectious diseases. However, these approaches are labor- and time-consuming, which does not allow for rapid diagnosis. Moreover, staff in the clinical microbiology department require specialized training. Currently, body fluid biomarkers and some novel laboratory technologies (e.g., nucleic acid amplification test (NAAT) and mass spectrum) are widely used in the clinical laboratory setting, and represent the major approaches for the diagnosis of infectious diseases. In this special series of Annals of Translational Medicine, experienced clinicians, microbiologists, and laboratory technicians contribute some of their valuable works in the field of laboratory testing of infectious diseases.

Body fluid biomarkers represent rapid, objective, and easily obtainable tools for infectious disease diagnosis. In this special series, two reviews discuss the diagnostic utility of biomarkers for community-acquired pneumonia (CAP) and tuberculosis pleural effusion (TPE). Leukocyte count, procalcitonin (PCT) and C-reactive protein (CRP) are three widely used laboratory tests for CAP; however, their diagnostic accuracy is far from perfect. In recent years, many novel diagnostic biomarkers for CAP have been identified, such as soluble triggering receptor expressed on myeloid cells-1 (sTREM-1), pro-adrenomedullin (proADM), and presepsin. The diagnostic utility of these markers has been reviewed in depth by Ito et.al. In another review, pleural biomarkers for TPE diagnosis were summarized, and it was concluded that adenosine deaminase (ADA) is the first choice for TPE diagnosis because of its high diagnostic accuracy, short turnaround time (TAT), and low cost. While pleural effusion interferon-gamma and interleukin-27 (IL-27) are also attractive because of their high diagnostic accuracy. Their high cost and non-standardized forms pose major disadvantages.

In this special series, two articles discuss the clinical application of novel laboratory technology for infectious disease diagnosis. In one work, a fast and novel method combining MALDI-TOF MS and UF-5000i urine flow cytometry was used to directly identify pathogenic bacteria in urine samples, reducing clinical work by at least one day and providing a reliable and efficient etiology basis for the timely diagnosis of UTIs. For urinary tract infections caused by single microorganisms, the identification coincidence rate was as high as $94.7 \%$ (249/263). In another review, the clinical utility of automated hematological analyzer for sepsis diagnosis was explored. Some novel parameters, such as VCS parameter, are now available in modern hematological analyzers and represent a rapid, objective and inexpensive tool for the diagnosis of sepsis.

In testing for infectious diseases, diagnostic accuracy and health benefit are two issues that need to be a considered, and the latter is more attractive for clinicians. In this special series, Azzini et al. reviewed the clinical utility of procalcitonin (PCT) in infectious disease. Notably, PCT is a "star" biomarker in infectious disease, owing to the accumulating bank of evidence that indicates that the PCT-guided antibiotics discontinuation algorithm is associated with lower mortality, shorter antibiotic duration, and fewer antibiotic-related side effects. In another study, the author analyzed the benefit of routine urine parameters for urine tract infection (UTI) screening. Some previous studies have evaluated the screening performance of routine urine parameters for UTI screening; however, this is the first study to investigate the net benefit of routine urine parameters. Despite the area under curve (AUC) of urine leukocyte count being statistically significant, they found that its net benefit is limited, while the net benefit of urine bacterial count is moderate.

Other articles included explore topics such as the molecular epidemiology of carbapenem-resistant Klebsiella Pneumonia 
(CRKP) and hypervirulence Listeria Monocytogenes, diagnostic assays for buman metapneumovirus, a technical improvement for HBsAg quantitation, and susceptibility testing on CHROMID ESBL ${ }^{\circledR}$ medium. Every one of these articles introduces rapid and accurate diagnostic methods, which may facilitate the future management of infectious diseases.

\section{Acknowledgements}

Funding: None.

\section{Footnote}

Provenance and Peer Review: This article was commissioned by the editorial office, Annals of Translational Medicine for the series "Advances in laboratory tests for infectious diseases". The article did not undergo external peer review.

Conflicts of Interest: Both authors have completed the ICMJE uniform disclosure form (available at http://dx.doi.org/10.21037/ atm-2020-id-13). The series "Advances in laboratory tests for infectious diseases" was commissioned by the editorial office without any funding or sponsorship. ZDH served as the unpaid Guest Editor of the series and serves as an unpaid Executive Editor of Annals of Translational Medicine from Apr 2020 to Mar 2025. BG served as the unpaid Guest Editor of the series and serves as an unpaid Managing Editor of Annals of Translational Medicine. The authors have no other conflicts of interest to declare.

Ethical Statement: The authors are accountable for all aspects of the work in ensuring that questions related to the accuracy or integrity of any part of the work are appropriately investigated and resolved.

Open Access Statement: This is an Open Access article distributed in accordance with the Creative Commons AttributionNonCommercial-NoDerivs 4.0 International License (CC BY-NC-ND 4.0), which permits the non-commercial replication and distribution of the article with the strict proviso that no changes or edits are made and the original work is properly cited (including links to both the formal publication through the relevant DOI and the license). See: https://creativecommons.org/licenses/by-nc$\mathrm{nd} / 4.0 \%$.

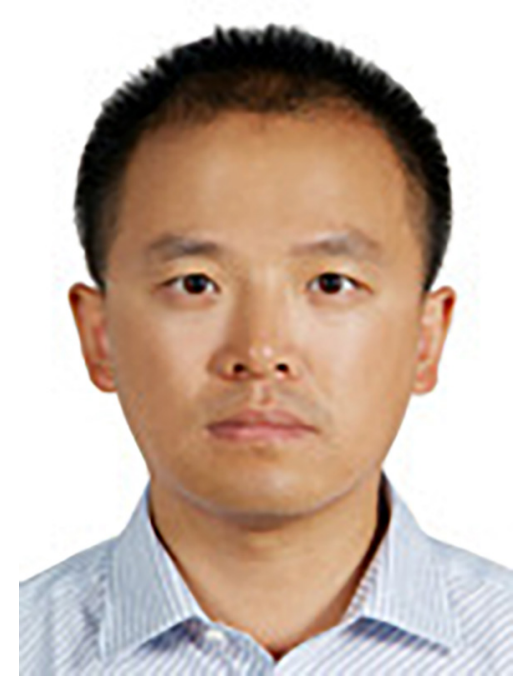

Zhi-De Hu

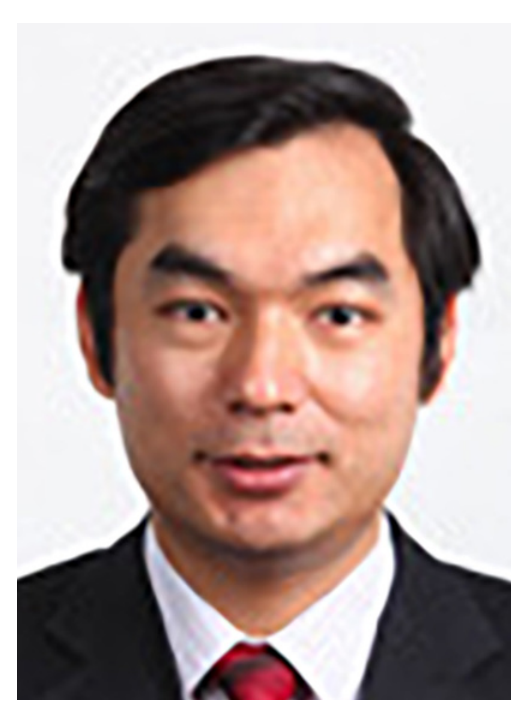

Bing Gu 
Zhi-De $\mathrm{Hu}^{1}$

${ }^{1}$ Department of Laboratory Medicine, The Affiliated Hospital of Inner Mongolia Medical University, Hobhot 010050, China

(Email: bzdlj81@163.com)

Bing $\mathbf{G u}^{2,3}$

${ }^{2}$ Medical Technology School of Xuzhou Medical University, Xuzhou 221004, China; ${ }^{3}$ Department of Laboratory Medicine, Affiliated Hospital of Xuzhou Medical University, Xuzhou 221002, China (Email: gb20031129@163.com) Submitted Apr 17, 2020. Accepted for publication Apr 27, 2020. doi: $10.21037 / \mathrm{atm}-2020$-id-13

View this article at: at http://dx.doi.org/10.21037/atm-2020-id-13

Cite this article as: $\mathrm{Hu} \mathrm{ZD}, \mathrm{Gu}$ B. Laboratory diagnosis of infectious diseases: a call for more rapid and accurate methods. Ann Transl Med 2020;8(9):600. doi: 10.21037/atm-2020-id-13 